\title{
Prognostic value of motor evoked potentials elicited by multipulse magnetic stimulation in a surgically induced transitory lesion of the supplementary motor area: a case report
}

\author{
F Sala, M J Krzan, G Jallo, F J Epstein, V Deletis
}

\begin{abstract}
Surgery involving the supplementary motor area (SMA) places the patient at risk of transient motor deficit. To predict outcome in patients with early postoperative hypokinesis would be relevant to both the patient and the surgical team. A 15 year old girl with a large left thalamic tumour removed through a left transcallosal approach is described. Despite intraoperatively preserved muscle motor evoked potentials (mMEPs) from all limbs, elicited by multipulse electrical stimulation, she awoke with a right hemiplegia and mutism. On the first postoperative day, neurophysiological evaluation using a multipulse magnetic stimulation technique, with a train of four magnetic stimuli, confirmed the presence of mMEPs from the hemiplegic right limbs. Slight spontaneous motor activity of the right limbs and initial speech were seen later on the same day with dramatic improvement over subsequent days.

It is concluded that multiple rather than single magnetic stimulation techniques may be needed to elicit mMEPs for an early postoperative differential diagnosis of SMA damage versus injury to the primary motor cortex or the corticospinal tract.

(F Neurol Neurosurg Psychiatry 2000;69:828-831)
\end{abstract}

Keywords: motor evoked potentials; multipulse magnetic stimulation; neurophysiological monitoring; supplementary motor area; brain tumour

Electrical stimulation of the exposed human cortex $^{1-3}$ and studies of cerebral metabolism measuring the regional cerebral blood flow ${ }^{4}$ support the important role the supplementary motor area (SMA) has in planning, initiation and control of motor tasks, and speech expression.

Fifty years ago, Penfield and Welsh ${ }^{1}$ noted that, although the most frequent effect of direct electrical stimulation of the SMA was inhibition of voluntary motor and speech activity, the unilateral excision of the SMA did not produce a permanent motor deficit. However, in the first few days after surgery, when the SMA syndrome is complete, hypokinesia can be so severe as to resemble the clinical picture of hemiplegia. Spontaneous motor activity then gradually recovers and long lasting motor deficits are usually limited to abnormal alternating movements of hands. ${ }^{5-8}$ Early after surgery, however, prognostic evaluation remains challenging because the clinical examination can not differentiate among SMA lesions and injury to the adjacent $M 1$ and corticospinal tract. Other authors ${ }^{8}$ attempted to use a single magnetic stimulation technique postoperatively to predict the outcome in patients with potential SMA syndrome after resective surgery, but they failed to elicit muscle motor evoked potentials (mMEPs) before clinical evidence of motor recovery.

We report on a patient with an SMA syndrome in whom postoperative mMEPs, elicited by multipulse (MMS) rather than single magnetic stimulation, gave evidence of preservation of $\mathrm{M} 1$ and corticospinal tract. These neurophysiological data ultimately correlated with prompt motor recovery despite initial hemiplegia and mutism.

\section{Case report}

A 15 year old right handed girl presented with a 2 month history of progressive right hemiparesis. Magnetic resonance imaging disclosed a large left heterogeneously enhancing thalamic tumour, with minimal adjacent oedema but moderate mass effect on the adjacent third and lateral ventricles (fig $1 \mathrm{~A}$ ).

The preoperative neurological examination showed a mild right hemiparesis, predominant in distal muscles of the upper limb, including a mild right central facial paresis. Hyperreflexia and a positive Babinski's sign on the right side were noted. Muscle tone was normal. Speech was not affected and there were no other neurological deficits. Under general anaesthesia the tumour was gross-totally resected through a left transcallosal approach with continuous neurophysiological monitoring. An external ventricular drain was placed at the end of surgery. 

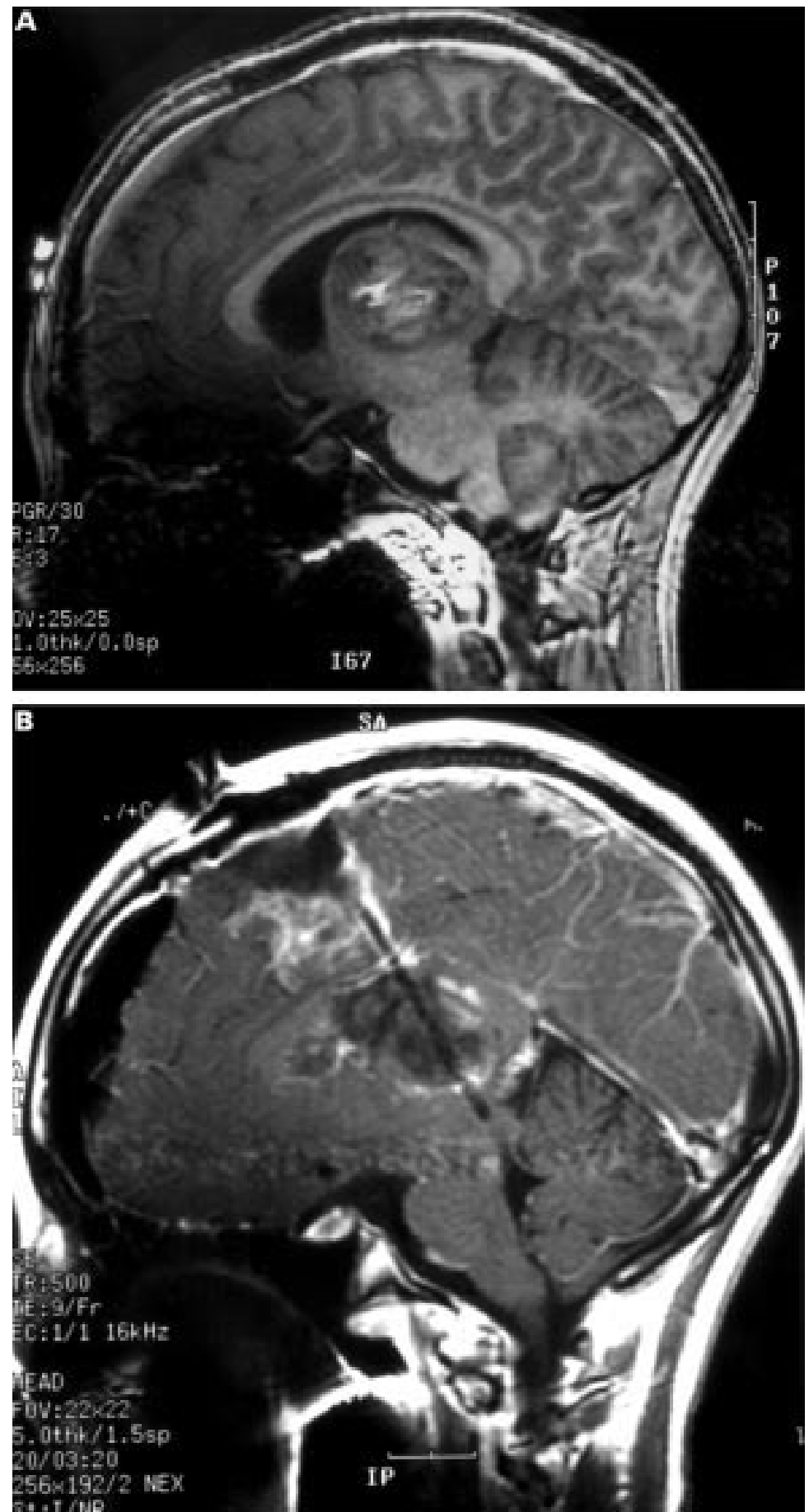

Figure 1 (A) Preoperative sagittal image showing a heterogeneously enhancing left thalamic tumour. (B) Postoperative sagittal enhanced T1 weighted MR images showing a gross total removal of the tumour through a left transcallosal approach, with signal abnormalities in the left frontomesial region.

INTRAOPERATIVE NEUROPHYSIOLOGICAL EVALUATION

Materials and methods

Cortical and subcortical somatosensory evoked potentials (CSEPs) were monitored as described elsewhere. ${ }^{9}$

Muscle MEPs were elicited by transcranial multiple electrical stimulation (MES) via corkscrew-like electrodes from the scalp (CS electrode, Neuromedical Inc, Herndon, VA, USA). Short trains of up to seven square wave stimuli of $500 \mu$ s duration and interstimulus intervals of $4 \mathrm{~ms}$ were applied at a repetition rate up to $2 \mathrm{~Hz}$ through electrodes placed at $\mathrm{C} 1$ and C2 scalp sites, according to the 10/20 EEG system. It has been previously shown that this montage activates the corticospinal tract for all four limbs. ${ }^{10}$ The stimulation intensity did not exceed $185 \mathrm{~mA}$. Muscle responses were recorded via needle electrodes inserted into the abductor pollicis brevis and tibialis anterior muscles bilaterally, and in the flexor digitorum superficialis and biceps brachii on the right side.

The Axon Sentinel-4 evoked potential system with modified software (AXON Systems, Inc, Hauppage, NY, USA) was used for stimulation and recording.

Once the dura was open, a grid electrode was placed on the exposed motor cortex and the median nerve CSEP phase reversal technique was used to identify the central sulcus. ${ }^{11}$ The grid electrode showing the phase reversed potential identifying the motor cortex was then used for direct stimulation of the right hand motor area. This allowed recording of mMEP responses from the right hand muscles using the same parameters of stimulation as for the transcranial technique of stimulation, except for much lower intensity $(10 \mathrm{~mA}) \cdot{ }^{12}$ Because the grid electrode was placed over the right hand area, mMEPs from other limbs were elicited by transcranial MES.

\section{Results}

During the surgical procedure the right and left median and tibial nerve CSEPs remained unchanged. Muscle MEP responses from the right as well as from the left limbs were present at the end of surgery; the response from the right tibialis anterior was temporarily lost during removal of the deepest part of the tumour but recovered by the end of the procedure. Clinical outcome-Unexpectedly, the patient woke up with a right hemiplegia and mutism. During the first 24 hours after surgery, she remained afebrile and alert, her mental status appeared intact, but mutism and right hemiplegia did not improve; no sedating medications were given. Twenty four hours after surgery MR imaging showed a gross-total tumour removal $(>95 \%)$ with moderate oedema in the left posteromesial frontal region. There was no significant oedema in the thalamic region and neither haemorrhage nor hydrocephalus was present (fig $1 \mathrm{~B}$ ). At this point, on the first postoperative day, the neurophysiological evaluation was repeated.

POSTOPERATIVE NEUROPHYSIOLOGICAL EVALUATION

Materials and methods

To avoid discomfort to the awake patient, mMEPs were tested in response to MMS (Quadropulse, MagStim Co, Ltd, Spring Gardens, Dyfed, UK). A train of four stimuli, with interstimulus intervals of $4 \mathrm{~ms}$ and intensity up to $70 \%$ of the maximal stimulator output was used. For focal stimulation we used a butterfly shaped coil, which was centred over the hand and leg areas of the motor cortex. This coil orientation induces current in the brain in the 


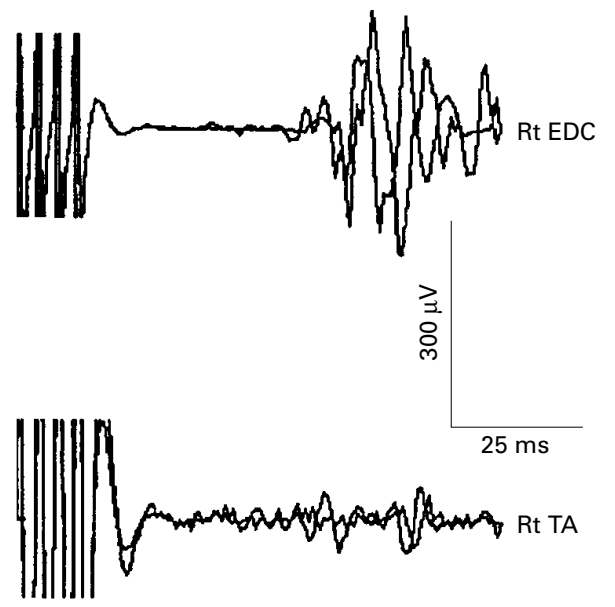

Figure 2 Presence of $m M E P$ s from right limbs despite right hemiplegia. MEPs were elicited on the first postoperative day by MMS using a short train of four stimuli with interstimulus intervals of $4 \mathrm{~ms}$. Note: the longer latencies of MEPs are a result of eliciting them with the multiple magnetic stimulation technique.EDC $=$ Extensor digitorum communis; $T A=$ tibialis anterior.

anteroposterior direction. Muscle MEPs were recorded percutaneously from limb muscles by cup-like EEG electrodes.

Results

The stimulation was repeated a few times, obtaining reproducibility of the responses elicited from the right extensor digitorum communis and right tibialis anterior (fig 2). CSEPs from all four limbs were unchanged compared with the recordings at the end of surgery.

Clinical outcome-On the same day, after mMEPs were recorded, the patient started to move her right limbs and uttered several words. Spontaneous motor activity and speech dramatically improved over the next few days. At discharge, 1 week later, only a mild right hemiparesis and dysnomia were present. The patient returned to school and at follow up 3 months later had only the same mild right hemiparesis seen before surgery.

\section{Discussion}

This report raises the issue of a patient who, despite the preservation of intraoperative mMEPs, emerged from anaesthesia with hemiplegia and mutism. Unexpected postoperative neurological deficits are traumatic to both patients, their family, and the surgical team. In this setting, the ability to predict outcome significantly contributes to the clinical management of these patients. Whenever neuroimaging studies are not conclusive, functionalrather than morphological-evaluation of the integrity of M1/CST using neurophysiological tests could be useful.

Zentner et $a l^{8}$ described 13 patients who had resective procedures involving the SMA. Despite preservation of mMEPs at the end of surgery in all of them, five patients emerged from anaesthesia with a typical SMA syndrome including hemiplegia contralateral to the resection side. Elicitability of mMEPs was tested early postoperatively using the single magnetic stimulus technique, but mMEPs were not present in the immediate postoperative period and reappeared only in the subsequent days, on a course parallel to that of clinical recovery. Accordingly, these findings raised questions on the prognostic value of mMEPs elicited by a single magnetic stimulus in the early postoperative period.

By contrast, in the patient we described, mMEPs from clinically plegic limbs were obtained 24 hours after surgery before any sign of clinical recovery. We hypothesise that the multipulse magnetic stimulation technique accounted for the different result from the single magnetic stimulation technique used by Zentner $e t a l,{ }^{8}$ which did not elicit mMEPs on the first postoperative day.

Mechanisms that facilitate the recording of mMEPs elicited by MMS are not clear yet. Magnetic stimulation of the cerebral cortex activates motoneurons predominantly transsynaptically, with induced current within the brain in an anterior-posterior direction. ${ }^{13}$ If an SMA lesion temporarily decreases the excitability of M1, single magnetic stimulation may not bring the excitatory postsynaptic potentials of cortical motor neurons to the firing threshold. In our experience, MMS is more effective than a single stimulus and requires a lower output intensity. ${ }^{14}$ However, the hypothesis of a transient hypoexcitability of the M1 secondary to an SMA lesion is not supported by experimental studies in monkeys where cooling of the SMA did not interfere with the neuronal discharge patterns in $\mathrm{M} 1 .^{15}$ Furthermore, neuroanatomical tracers injected in the SMA disclosed the existence of corticospinal axons labelled anterogradely all the way down to the spinal cord. ${ }^{16}$ The study suggested that the SMA directly influences the motoneurons and likely modulates motor pathway excitability also at the spinal cord level.

In the patients presented by Zentner et $a l^{8}$ the reappearance of mMEPs in the subsequent days might be explained by the compensatory part played by the contralateral SMA. This compensatory mechanism, however, is very unlikely in patients with bilateral lesions of the SMA, where mMEPs might not correlate with functional outcome. ${ }^{17}$

In our patient we noted a faster motor and speech recovery than that described in the classic SMA syndrome where a few weeks are usually required to return to baseline motor function and normal speech. ${ }^{6-8}$ In the past, similar cases raised questions whether these deficits are the result of an SMA injury itself rather than being secondary to a "surgical shock" affecting the adjacent areas, including the M1/CST. ${ }^{57}$ In our case, considering the surgical route to the deep thalamic lesion, as depicted by the postoperative MRI, a postoperative oedema secondary to hemispheric retraction or disruption of cortical vessels may have caused "surgical shock". Whereas the possibility of a transitory complete motor deficit despite preserved mMEPs has been reported in patients with stroke, ${ }^{18}$ we are not aware of any report of normal mMEPs and hemiplegia due to a surgically induced M1/ CST lesion. Therefore, if "surgical shock" was 
indeed the explanation for the syndrome we found, this foremost affected the SMA, not the M1/CST.

In this patient, neurophysiological testing with postoperative MMS predicted recovery only a few hours before motor deficits began to improve. However, in patients with SMA syndrome akinesia may last for several days ${ }^{19}$ and MMS would therefore retain a significant prognostic role.

Finally, whereas the tumour location was not typical for those expected to produce a true SMA syndrome based on their resection, we suggest that the transcallosal approach may have injured the SMA or its subcortical projections causing a reversible SMA injury. Based on the presence of preoperative motor deficit, the possibility that either frontal premotor areas or the thalamic region could have been temporarily affected cannot be ruled out, but it was not supported by postoperative MR imaging. Even in this scenario, the postoperative integrity of M1/CST documented by MMS would have suggested a good motor outcome.

\section{Conclusion}

In patients with severe motor deficit in the immediate postoperative period, despite preserved intraoperative $\mathrm{mMEPs}$, diagnosis and prognosis are challenging. In this report, mMEPs elicited by MMS in clinically hemiplegic limbs correctly predicted good motor outcome. This technique could have a significant role in the assessment of the functional integrity of motor pathways in cases of unexpected postoperative paralysis such as those secondary to SMA syndromes.

We thank Dr Markus Kofler for assistance and suggestions in preparing this manuscript and Marco Campos for his editorial help.

1 Penfield W, Welch K. The supplementary motor area of the cerebral cortex: a clinical and experimental study. Arch Neurol Psychiatry 1951;66:289-317.

2 Lim SH, Dinner DS, Pillay PK, et al. Functional anatomy of the human supplementary sensorimotor area: results of extraoperative electrical stimulation. Electroencephalogr Clin Neurophysiol 1994;91:179-93.
3 Ikeda A, Luders HO, Burgess RC, et al. Movement-related potentials recorded from supplementary motor area and
primary motor area. Role of supplementary motor area in primary motor area. Role of supplementary mot

4 Roland PE, Larsen B, Lassen NA, et al. Supplementary motor area and other cortical areas in organization of voluntary movements in man. $\mathcal{F}$ Neurophysiol 1980;43:11836.

5 Bleasel A, Comair Y, Luders HO. Surgical ablations of the mesial frontal lobe in humans. In: Luders $\mathrm{HO}$, ed. Advances in neurology. Vol 70. Supplementary sensorimotor area. Philadelphia: Lippincott-Raven, 1996:217-35.

6 Laplane D, Talairach J, Meininger V, et al. Clinical consequences of corticectomies involving the supplementary motor area in man. $\mathcal{F}$ Neurol Sci 1977;34:301-14.

7 Rostomily RC, Berger MS, Ojemann GA, et al. Postoperative deficits and functional recovery following removal of tumors involving the dominant hemisphere supplementary motor area. $\mathcal{F}$ Neurosurg 1991;75:62-8.

8 Zentner J, Hufnagel A, Pechstein U, et al. Functional results after resective procedures involving the supplementary motor area. F Neurosurg 1996;85:542-9.

9 Deletis V, Engler GL. Somatosensory evoked potentials for spinal cord monitoring. In: Bridwell Kh, DeWald RL, eds. The textbook of spinal surgery. Philadelphia: LippincottRaven, 1997:85-92.

10 Kothbauer K, Deletis V, Epstein FJ. Motor-evoked potential monitoring for intramedullary spinal cord tumor surgery: correlation of clinical and neurophysiological data in a series of 100 consecutive procedures. Neurosurg Focus 1998;4:1.

11 Cedzich C, Taniguchi M, Schäfer S, et al. Somatosensory evoked potentials phase reversal and direct motor cortex stimulation during surgery in and around the central stimulation during surgery in and
region. Neurosurgery 1996;38:962-70.

12 Pechstein U, Cedzich C, Nadstawek J, et al. Transcranial high-frequency repetitive electrical stimulation of recording myogenic motor evoked potential with the patient under general anesthesia. Neurosurgery 1996;39:335-44.

13 Kaneko K, Kawai S, Fuchigami Y, et al. The effect of current direction induced by transcranial magnetic stimulation on the corticospinal excitability in human brain. Electroencephalogr Clin Neurophysiol 1996;101:478-82.

14 Deletis V, Kothbauer K. Intraoperative neurophysiology of the corticospinal tract. In: Stölberg E, Sharma HS, Olsson $\mathrm{Y}$, eds. Spinal cord monitoring. Vienna: Springer-Verlag, 1998:421-44.

15 Schmidt EM, Porter R, McIntosh JS. The effects of cooling supplementary motor area and midline cerebral cortex on neuronal responses in area 4 of monkeys. Electroencephalogr Clin Neurophysiol 1992;85:61-71.

16 Rouiller EM. Multiple hand representations in the motor cortical areas. In: Wing AM, Haggard P, Flanagan JR, eds. Hand and brain: the neurophysiology and psychology of hand movements. San Diego: Academic Press, 1996:99-124.

17 Kofler M, Morota N, Deletis V. Preserved motor evoked potentials fail to predict functional outcome in quadriplegia because of bilateral lesions of the supplementary motor areas: a brief report. Am f Phys Med Rehabil 1999;78:6671 .

18 Rapisarda G, Bastings E, Maertens de Noorhout A, et al. Can motor recovery in stroke patients be predicted by early transcranial magnetic stimulation? Stroke 1996;27:2191-6.

19 Schell G, Hodge CJ, Cacayorin E. Transient neurological deficit after therapeutic embolization of the arteries supplying the medial wall of the hemisphere, including the supplementary motor area. Neurosurgery 1986;18:353-6. 